\title{
Total laparoscopic hysterectomy versus total abdominal hysterectomy with bilateral salpingo-oophorectomy for endometrial carcinoma: a randomised controlled trial with 5-year follow-up
}

\author{
Kirsten B. Kluivers • Florien A. Ten Cate • \\ Marlies Y. Bongers • Hans A. M. Brölmann • \\ Jan C. M. Hendriks
}

Received: 30 August 2010 / Accepted: 13 February 2011 /Published online: 11 March 2011

(C) The Author(s) 2011. This article is published with open access at Springerlink.com

\begin{abstract}
This report is on recovery and long-term outcomes in a small-scale randomised controlled trial (RCT) after total laparoscopic hysterectomy versus total abdominal hysterectomy in (potential) endometrial carcinoma patients. An RCT was performed among women with atypical endometrial hyperplasia and endometrial carcinoma scheduled for hysterectomy in a teaching hospital in The Netherlands. Women were randomised to total laparoscopic hysterectomy versus total abdominal hysterectomy both with bilateral salpingo-oophorectomy and were followed until 5 years after the intervention. Patients completed the RAND 36-Item Short Form Health Survey (RAND-36), Quality of Recovery-40 (QoR-40) and Recovery Index-10 (RI-10) until 12 weeks
\end{abstract}

K. B. Kluivers $(\bowtie)$

Department of Obstetrics \& Gynaecology,

Radboud University Nijmegen Medical Centre,

PO Box 9101, 6500 HB Nijmegen, The Netherlands

e-mail: K.Kluivers@obgyn.umcn.nl

F. A. Ten Cate $\cdot$ M. Y. Bongers

Department of Obstetrics \& Gynaecology,

Máxima Medical Centre,

De Run 4600,

5504 DB Veldhoven, The Netherlands

\section{H. A. M. Brölmann}

Department of Obstetrics \& Gynaecology,

VU University Medical Centre,

De Boelelaan 1117,

1081 HV Amsterdam, The Netherlands

J. C. M. Hendriks

Department of Epidemiology and Biostatistics,

Radboud University Nijmegen Medical Centre,

PO Box 9101, 6500 HB Nijmegen, The Netherlands after surgery. Main outcome measure was quality of life and recovery in the first 12 weeks after surgery. A linear mixed model was used for statistical analysis while accounting for baseline values where applicable. Seventeen women were included, of whom 11 allocated to the laparoscopic arm and 6 to the abdominal arm. Laparoscopic hysterectomy performed better on all scales and subscales used in the study. A statistically significant treatment effect, favouring laparoscopic hysterectomy, was found in the total RAND-36 (difference between groups 142 units, 95\% confidence interval 46; 236). Clinical follow-up was completed after median 60 months, but this study was too small for conclusions regarding the safety and survival. Laparoscopic hysterectomy results in better postoperative quality of life in the first 12 weeks after surgery when compared with abdominal hysterectomy.

Keywords Abdominal hysterectomy - Endometrial carcinoma $\cdot$ Laparoscopic hysterectomy · Randomised . Recovery $\cdot$ Quality of life

\section{Background}

Different approaches to hysterectomy have been extensively studied for the benign indications, and randomised controlled trials (RCTs) have been summarized in systematic review and meta-analysis [1]. One of the important results was a significant 2.6-fold increased risk of urinary tract injury. Recovery and quality of life were shown to be better after laparoscopic hysterectomy [2, 3]. In malignant disease, 
however, clinical outcomes such as complication rates or postoperative recovery may be different from the benign indications. The women are generally older, and the uterus is mostly smaller and less vascularised. And recurrence rate and survival rate are probably the most important long-term outcomes in these women.

At this moment, there are eight RCTs [4-12] available comparing laparoscopic and abdominal hysterectomy in (potential) endometrial carcinoma patients. Four RCTs [47] have been summarized in a systematic review in 2008 [13] and four further RCTs are available since then [8-12].

Quality of life was an outcome measure in four studies on 1,526 women $[5,9-11]$. The laparoscopic approach was favourable in all studies, although this difference proved to be not clinically relevant in one study [9]. Better quality-oflife scores were found up till 3 months in one study [10] and up till 6 months after surgery in two further studies $[5,11]$.

Only two study groups on previous RCTs have already analysed the recurrence and survival rates by treatment group with 3 [4] and 6 years [12] median follow-up. These studies showed similar rates after laparoscopic and abdominal hysterectomy among 206 women. Data on recurrence and survival from the three large RCTs that have recently closed the inclusion on another 1,442 women are awaited with interest [9-11]. Future systematic review and meta-analysis of RCTs will provide a reliable answer to the question whether laparoscopic hysterectomy is a safe approach in women with endometrial cancer.

In the present paper, the results of a small RCT comparing total laparoscopic hysterectomy and total abdominal hysterectomy in women with atypical endometrial hyperplasia and endometrial carcinoma are presented. Postoperative quality of life as measured by the RAND36 health survey and long-term clinical outcomes, including recurrence rates until 5 years after the intervention, have been assessed.

\section{Methods}

\section{Participants}

This study was performed in the Maxima Medical Centre, a large teaching hospital in the south of The Netherlands, from August 2002 through January 2005. The gynaecologic department is experienced in minimal invasive surgery, with 10 years experience in laparoscopic hysterectomy before the start of the study.

Patients were eligible for the study in case there was no suspicion of endometrial carcinoma beyond FIGO stage 1, and the size of the uterus did not exceed 18 weeks of gestation. Chest X-ray was performed in all women, but no routine hysteroscopy was performed for preoperative staging. Exclusion criteria were a previous lower abdominal midline incision, the need for simultaneous interventions and inability to speak Dutch. Approval for the study was obtained from the ethics committee of the Maxima Medical Centre on July 25, 2002 under number 0217. There is no overlap of the population with other published Dutch studies $[3,10]$. Written informed consent was required for participation. The study was performed in line with the CONSORT statement.

Peritoneal fluid or cytological washing from the abdominal cavity was sent for pathology, but no standard peritoneal biopsies or lymphadenectomy was carried out in either group. No frozen sections were planned or performed. The laparoscopic procedures were total laparoscopic hysterectomies (TLH), where the operation of the vaginal part was restricted to the removal of the laparoscopically freed uterus, and the vaginal vault was sutured laparoscopically [2]. In laparoscopic hysterectomy, the uterus was manipulated by the Karl Storz Clermont-Ferrand manipulator (Karl Storz, Tuttlingen, Germany) after cauterization of the fallopian tubes to avoid spillage of cancer cells. In abdominal hysterectomy, the uterus was manipulated with the use of two Heaney clamps placed over the fallopian tube-ovarian ligament complex on both sides. There was an equal dissection of pelvic tissues in the laparoscopic as compared with the open technique.

The primary outcome of this study was quality of life. Three questionnaires have been used in the study: the RAND-36 health survey (RAND-36), Quality of Recovery40 (QoR-40) and Recovery Index-10 (RI-10). The three questionnaires have previously been validated for the Dutch language $[14,15]$. The RAND-36 is a generic health-related quality-of-life questionnaire. The questionnaire measures subjective health in eight scales, which range from 0 to 100 . Thus, the total score ranges from 0 to 800 , where 0 is the poorest quality of life and 800 the best imaginable. Summated ratings and standardized scoring algorithms were used to assess the eight scales [14]. The QoR-40 and RI-10 are postoperative recovery-specific questionnaires [15]. The QoR-40 consists of 40 items in five subscales: emotional state, physical comfort, psychological support, physical independence and pain. Each item was answered on a five-point Likert scale, ranging from none of the time to all the time. The QoR-40 score was defined as the sum of the scores of all items. The QoR-40 score ranged from 40 to 200 , in which 200 indicates a perfect recovery. The items referred to the past $24 \mathrm{~h}$ and aimed at patients during hospital stay, but could be filled out at home as well. The RI-10 is a ten-item questionnaire measuring postoperative recovery on five-point Likert scales ranging from full disagreement to full agreement. The instrument has no separate subscales, and the score ranges from 10 to 50 , 
where 50 indicates a perfect recovery. The items referred to the past week. Since most of the items in the RI-10 referred to the postoperative situation, no baseline measurement was available. The items suited best for recently discharged patients, but could be filled out in hospital as well.

Patients filled out the questionnaires without assistance of the researcher and returned the questionnaires by mail. The baseline measurement was completed after randomisation. Measurement moments were as proposed for surgical studies in the previous validation study [15].

The surgical outcome (peri and postoperative clinical outcome) and oncological outcome (recurrence and survival rates) were secondary outcome measures of the study. FIGO nomenclature (Rio de Janeiro 1988) was used to describe surgical cancer stages. The decision for postoperative external beam pelvic radiotherapy was made as based on the PORTEC trial [16]. Data were collected by completion of a standardized case record form. At the end of follow-up in the present study (June 2008), all patients were interviewed on any adverse outcomes or events from the condition or surgery. In case no written reply was received, telephone calls were made to complete these data.

\section{Sample size and randomisation}

The study has been performed parallel to a larger study on laparoscopic versus abdominal hysterectomy in benign conditions [2]. For that study, the sample size was calculated for the quality of life as measured by the questionnaire RAND36. A difference of 15 per scale was considered as clinically relevant. With a standard deviation of 20, a type I error of 0.05 , and $80 \%$ power, 28 patients were needed per arm.

After obtaining written informed consent, randomisation took place by opening numbered, sealed opaque envelopes. For concealment, an independent person had randomly assigned an equal number of 38 papers with either intervention to the envelopes. The closed envelopes were shuffled before numbering, and were used for both patients with benign and malignant disease. The patients and medical team were not blinded to the intervention.

\section{Statistical methods}

The data are presented on intention-to-treat basis. The medians with the range are presented in case of continuous data and absolute numbers with percentages in case of dichotomous variables. Differences in medical outcome between the two treatment groups were tested for statistical significance using the Mann-Whitney $U$ test in case of continuous data, and the Fisher's exact test in case of two by two tables. A linear mixed model was used to study the differences in scores on the questionnaires between the laparoscopic and abdominal group over time while accounting for the baseline values for each of the scales and subscales separately [17]. The dependent variable was the (sub)scale of RAND-36, QoR-40 or RI-10. The independent class variables were patient and treatment (laparoscopic and abdominal hysterectomy, respectively) and time since surgery, and the independent regression variables was the baseline level. Both the intercept and the regression in time of each patient were treated as random variables in the model. This way differences between treatments are estimated given the baseline value, while differences in recovery among patients are allowed. Initially, interaction terms and quadratic terms in time were included in the linear part of the model; but as the inclusion did not significantly (likelihood-ratio test) improve the fit to the data, these terms were not included in the final model used [17]. Note that excluding the interaction term of group with time, results in a parallel line model, (i.e. the differences between groups are identical at each point of measurement). The estimated regression parameters with standard errors of each score are used to calculate the average level per week of the patients in each group. These levels with confidence bands are further presented in figures.

The quality-of-life data were analysed by SAS 8.2 software (SAS Institute, Inc., Chicago, IL, USA), all other data in SPSS 16.0 software (SPSS, Inc., Chicago, IL, USA), with $p$ values $<0.05$ considered statistically significant.

\section{Findings}

Seventeen women were randomised, of whom 11 were allocated to the laparoscopic arm and 6 were allocated to the abdominal arm. Patient characteristics and surgical indications are shown in Table 1. A flowchart of the study is presented in Fig. 1. Overall median clinical follow-up after the operation was 60 months (range 18-81 months) until the last gynaecological examination. The interviews on any adverse outcomes were minimum 45 months after surgery (median 65 months, range $45-81$ months). No women were lost to follow-up.

The surgical and oncological outcome

Data on the procedures including histological findings are presented in Table 2. There was one intra-operative conversion from laparoscopy to laparotomy, which was related to difficult access due to adiposity and $300 \mathrm{~mL}$ blood loss during laparoscopy. Her recovery and followup were uneventful. All other laparoscopic hysterectomies were performed as TLH. There was no macroscopically visible or palpable tumour extra-uterine tumour in either group. All final histologies showed endometroid type adenocarcinoma, except in a woman with FIGO 
Table 1 Patient characteristics and surgical indications by treatment group

\begin{tabular}{lll}
\hline & LH $(n=11)$ & AH $(n=6)$ \\
\hline Age (years) & $59[49-69]$ & $64[59-73]$ \\
BMI $\left(\mathrm{kg} / \mathrm{m}^{2}\right)$ & $27[21-50]$ & $27[21-32]$ \\
Parity & $2[0-4]$ & $2[0-6]$ \\
ASA score & $2[1-3]$ & $1[1-2]$ \\
Uterine weight $(\mathrm{g})$ & $100[50-175]$ & $75[50-280]$ \\
Indication for surgery & & \\
Atypical hyperplasia & $3(27 \%)$ & $2(33 \%)$ \\
Endometrial carcinoma & $8(73 \%)$ & $4(66 \%)$ \\
Baseline RAND-36 & $581[366-744]$ & $670[278-726]$ \\
$\quad$ QoR-40 & $183[147-200]$ & $184[157-200]$ \\
$\quad$ RI-10 & n.a. & n.a. \\
\hline
\end{tabular}

Data presented as median [range] or absolute numbers (percentage)]

$A H$ abdominal hysterectomy, $A S A$ American Society of Anesthesiologists, $B M I$ body mass index, $L H$ laparoscopic hysterectomy, $R A N D$ 36 RAND 36-Item Short Form Health Survey, QoR-40 Quality of Recovery-40, RI-10 Recovery Index-10, n.a. not applicable

stage 3 a grade 2 in the laparoscopic hysterectomy group, who had a mixed-cell type tumour (endometroid and serous adenocarcinoma).

Complications by treatment group are presented in Table 3. In one patient, randomised to abdominal hysterectomy, final histology demonstrated that the cervix had not been removed completely in a woman with atypical endometrial hyperplasia. It has been decided not to perform a reoperation. Her follow-up has been uneventful, besides a tension-free vaginal tape (TVT)] procedure, which has been performed due to deterioration of stress urinary incontinence 2 years postoperatively. Other complications in the abdominal hysterectomy group were: 1,450 mL blood loss during surgery, one woman with temporary atrial flutter, one woman with temporary low oxygenation, a urinary tract infection and fever of unknown origin in the immediate postoperative period. One woman developed radiation colitis after radiotherapy.

In the laparoscopic hysterectomy group, two women died because of a recurrent endometrial carcinoma. Of these two women, one was a 57-year-old woman with Figo stage $1 \mathrm{c}$ grade 2 disease, and no postoperative radiotherapy had a locoregional recurrence 12 months after surgery and died 2 months later. A stent was inserted for bowel obstruction, but the extensiveness of the metastases and her general condition did not allow for any adjuvant therapy. The other was a 64-year-old woman with the mixed cell-type tumour stage 3 a grade 2 underwent pelvic radiotherapy and had a recurrence 11 months after surgery. Her general condition did not allow for

adjuvant chemotherapy, and she died 18 months after surgery. Other complications in the laparoscopic hysterectomy group were a urinary tract infection, deterioration of pre-operative micturition problems with longterm catheterization, back pain for which a neurologist was consulted but found no abnormalities. One woman developed radiation colitis after radiotherapy. No other complications (such as blood transfusions, visceral damage or port-site metastasis) occurred in either group.

\section{Quality of life and recovery}

Overall, only four questionnaires were missing, and thus, the return rate of the questionnaires was $97 \%$. Table 4 and Fig. 2a, b and c show the differences in total scores on the three questionnaires between laparoscopic and abdominal hysterectomy. No scale or subscale was in favour of abdominal hysterectomy. The difference between groups in

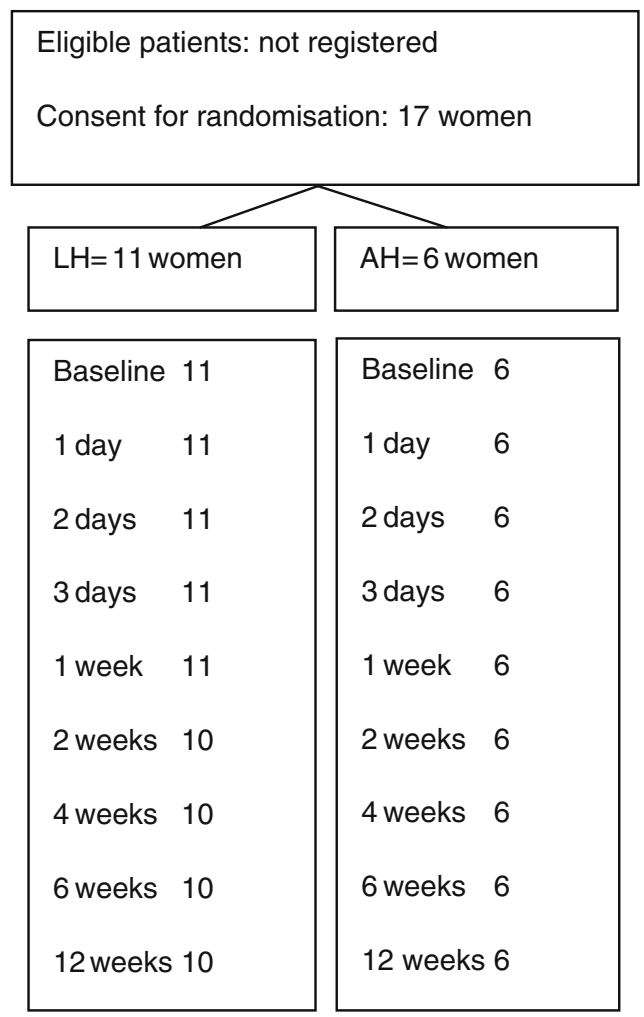

5 years:

Recurrence and deceased 2

Follow up complete

Fig. 1 Flowchart of the study 
Table 2 Data on surgical procedures and oncological outcome by treatment group

\begin{tabular}{llll}
\hline & LH $(n=11)$ & AH $(n=6)$ & $P$ value \\
\hline Intra-operative laparoconversion & $1(9 \%)$ & n.a. & n.a. \\
Operation time (min) & $122[96-168]$ & $80[70-192]$ & 0.03 \\
Blood loss (mL) & $200[0-550]$ & $350[300-1450]$ & 0.01 \\
Hospitalization (days) & $5[4-16]$ & $8[5-11]$ & $64[50-81]$ \\
Clinical follow-up (months) & $54[18-80]$ & 0 & 0.03 \\
Diseased after recurrence & $2(18 \%)$ & & 0.09 \\
Postoperative histology & $1(9 \%)$ & $1(17 \%)$ & 0.40 \\
Normal & 0 & $5(83 \%)$ \\
Atypical hyperplasia & $10(91 \%)$ & $3(50 \%)$ \\
Endometrial carcinoma & $3(27 \%)$ & 0.60 \\
Postoperative radiotherapy & & & 0.34 \\
\hline
\end{tabular}

Data shown as absolute numbers (percentage) or median [range]. $p=p$ value for differences between groups using Fisher's exact test in cases of 2by-2 tables, and Mann-Whitney test in cases of non-normal distributed numerical variables

AH abdominal hysterectomy, $L H$ laparoscopic hysterectomy, n.a. not applicable

${ }^{a}$ Endometrial carcinomas were stage $1 \mathrm{~b}$ grade 1 (2 patients), 1c grade 1, 2a grade 1, and 3a grade 1 in the AH group, and 1a grade 1, 1b grade 1 (3 patients), $1 \mathrm{~b}$ grade 2, $1 \mathrm{c}$ grade $1,1 \mathrm{c}$ grade 2 (2 patients), $2 \mathrm{~b}$ grade 1 and $3 \mathrm{a}$ grade 2 in the LH group. All histologies showed endometroid type adenocarcinoma, except one woman in the LH group with stage 3a grade 2 mixed cell-type tumour (endometroid and serous adenocarcinoma)

the RAND-36 total score was 142 units $(95 \%$ confidence interval (CI) 46; 236) in favour of laparoscopic hysterectomy. Furthermore, in three RAND-36 subscales relating to physical well-being, statistically significant treatment effects in favour of laparoscopic hysterectomy were found (data not shown). One QoR-40 subscale and the total RI-10 showed borderline significant differences. Note that the difference between the treatment groups are presented after correction for differences in baseline values between the groups. The estimated increase per 10 units higher level in the total RAND-36 score at baseline between two patients was $7(95 \% \mathrm{CI} 4$; 11) units. This was independent of both timepoint and treatment group. Furthermore, the estimated increase in the total RAND-36 score at 12 weeks after surgery compared with
1 week after surgery was $122(95 \%$ CI $67 ; 178)$ units in both groups.

\section{Conclusions}

In this RCT, the difference in recovery was assessed between total laparoscopic hysterectomy and total abdominal hysterectomy, both with bilateral salpingo-oophorectomy, in patients scheduled for hysterectomy for reason of atypical endometrial hyperplasia or endometrial carcinoma. After baseline correction, we report a statistically significant difference in postoperative quality of life as measured by RAND-36, favouring laparoscopic hysterectomy. This difference between the treatment groups was present all along the
Table 3 Complications by treatment group

$A H$ abdominal hysterectomy, $L H$ laparoscopic hysterectomy, n.a. not applicable

Data shown as absolute numbers (percentage). $p=p$ value for difference between groups using Fisher's exact test

${ }^{a}$ Deterioration stress urinary incontinence

${ }^{\mathrm{b}}$ TVT increased residual urine with self-catheterization

${ }^{\mathrm{c}}$ Note that three women in each group received radiotherapy

\begin{tabular}{llll}
\hline & LH $(n=11)$ & AH $(n=6)$ & $P$ value \\
\hline Blood loss $>1,000 \mathrm{~cm}^{3}$ & 0 & $1(17 \%)$ & 0.35 \\
Fever (unknown origin) & 0 & $1(17 \%)$ & 0.35 \\
Urinary tract infection & $1(9 \%)$ & $1(17 \%)$ & 1.0 \\
Cervical stump problems & 0 & $1(17 \%)$ & 0.35 \\
Urinary symptoms & $1(9 \%)^{\mathrm{a}}$ & $1(17 \%)^{\mathrm{b}}$ & 1.0 \\
Temporary low saturation & 0 & $1(17 \%)$ & 0.35 \\
Atrial flutter & 0 & $1(17 \%)$ & 0.35 \\
Back pain & $1(9 \%)$ & 0 & 1.0 \\
Radiation colitis & $1(33 \%)^{\mathrm{c}}$ & $1(33 \%)$ & 1.0 \\
Total complications & 4 & 8 & $\mathrm{c} . \mathrm{a}$. \\
No of patients with complications & $3(27 \%)$ & $5(83 \%)$ & 0.05 \\
\hline
\end{tabular}


Table 4 Estimated increase in questionnaire score level $(95 \%$ confidence interval) by treatment group, by unit increase in baseline score and point of measurement using a linear mixed model

\begin{tabular}{|c|c|c|c|}
\hline & RAND-36 & QoR-40 & RI-10 \\
\hline \multicolumn{4}{|c|}{ Treatment group ${ }^{\mathrm{a}}$} \\
\hline LH & $142(46 ; 236)$ & $9(-3 ; 21)$ & $7(0 ; 14)$ \\
\hline $\mathrm{AH}$ & 0 (reference) & 0 (reference) & 0 (reference) \\
\hline \multicolumn{4}{|l|}{ Baseline effect } \\
\hline per 10 units $^{\mathrm{b}}$ & $7(4 ; 11)$ & $5(1 ; 9)$ & n.a. \\
\hline \multicolumn{4}{|c|}{ Time after surgery } \\
\hline 1 day & n.a. & 0 (reference) & n.a. \\
\hline 2 days & n.a. & $4(-3 ; 12)$ & n.a. \\
\hline 3 days & n.a. & $4(-4 ; 11)$ & n.a. \\
\hline 1 week & 0 (reference) & $13(6 ; 20)$ & 0 (reference) \\
\hline 2 weeks & $-8(-64 ; 47)$ & $26(18 ; 33)$ & $0(-3 ; 2)$ \\
\hline 4 weeks & $49(-6 ; 105)$ & n.a. & $4(1 ; 6)$ \\
\hline 6 weeks & $130(74 ; 185)$ & n.a. & $6(4 ; 9)$ \\
\hline 12 weeks & $122(67 ; 178)$ & n.a. & $8(5 ; 11)$ \\
\hline
\end{tabular}

Example: On average, the estimated level of the total RAND-36 score in the LH group was 142 units higher as compared with the AH group, at each timepoint from 2 to 12 weeks after surgery. The estimated increase per 10 units higher level in the total RAND-36 score at baseline between two patients was 7 units. This was independent of both timepoint and treatment group. Furthermore, the estimated increase in the total RAND-36 score at 12 weeks after surgery, compared to 1 week after surgery, was 122 units in both groups

Because the data fit very well to the parallel-line model the differences over time after surgery is estimated to be identical in both groups (see also Fig. 2a, b and c)

$L H$ laparoscopic hysterectomy, $A H$ abdominal hysterectomy, $R A N D$ 36 RAND 36-Item Short Form Health Survey, QoR-40 Quality of Recovery-40, RI-10 Recovery Index-10, n.a. not applicable

${ }^{a}$ Difference between groups after correction for baseline differences

${ }^{b}$ The baseline effect is the increase in postoperative score per 10 units increase at baseline

recovery period until 12 weeks after surgery. All subscales of RAND-36 and QoR-40, as well as the RI-10, showed a favourable outcome after laparoscopic hysterectomy compared with abdominal hysterectomy. This is in agreement with other studies on quality of life after surgery in (potential) endometrial carcinoma patients [5,9-11] and our previous findings in a larger benign population [2]. We found that the laparoscopic procedure took longer to perform, but with regard to other surgical and oncological outcomes, the present study is limited by its small sample size. These data may however be useful in future meta-analysis on the topic.

Lymphadenectomy has not been performed in this study amongst women with clinical stage I disease with expected low-grade endometrial carcinoma based on endometrial biopsy. Recently, two RCTs have failed to demonstrate any advantage over routine lymphadenectomy in endometrial carcinoma patients [18]. Nonetheless, routine lymphadenectomy in endometrial carcinoma is still under debate [19]. It is common practice throughout the Netherlands to perform hysterectomy and bilateral salpingo-oophorectomy in these women and advice postoperative radiotherapy as based on the PORTEC studies $[16,20]$. Thus, the results from our trial are limited to the population undergoing this type of treatment. Furthermore, note that PORTEC-2 data were not yet available at the time of the study [21], and no vaginal brachytherapy has been applied in the study.

In hysterectomies performed for potential malignant indications, recurrence and survival rates are of utmost importance. Although, there are no indications that laparoscopy is less safe $[5,12]$, this has not definitively been proven until now. In the present study, two women in the laparoscopic arm had a recurrence and died due to their malignancy. One woman had a bad prognosis due to a highstage mixed cell tumour. In line with the PORTEC study, the other woman had not received postoperative radiotherapy to minimize her risk of locoregional recurrence. With a FIGO stage $1 \mathrm{c}$ grade 2 and aged 57 years, she was, however, rather close to the range of less favourable prognostic factors with indication for radiotherapy.

Not only the route of hysterectomy, e.g. abdominal or laparoscopic hysterectomy, may influence recurrence rates and all other potential factors should thus be reported in according studies. In the present study, for example, an intra-uterine manipulator has been used. The impact on outcomes as compared with, e.g. vaginal manipulation with a tube only is not known until now. In two previous studies on peritoneal washings before and after manipulation with an intra-uterine device, spread of malignant cells could be demonstrated in 2 out of 82 women with negative peritoneal washings prior to manipulation $[22,23]$. Only one of these two women has sufficiently long follow-up (28 months) to report that no recurrence of malignancy has occurred [23]. Another histological phenomenon, "vascular pseudo invasion" of tumour into the blood vessels, has been described after manipulation with an intra-uterine balloon causing high intra-uterine pressures. Since this may have consequences for the staging and further therapy, this phenomenon may be regarded as undesirable and awareness among pathologists is needed [24].

The finding that quality of life was better after laparoscopic hysterectomy goes hand in hand with a different spectrum of complications as compared with other types of hysterectomy. A meta-analysis on benign indications reported a significant 2.6-fold increased risk of urinary tract injury [1]. Whether this also applies to endometrial 

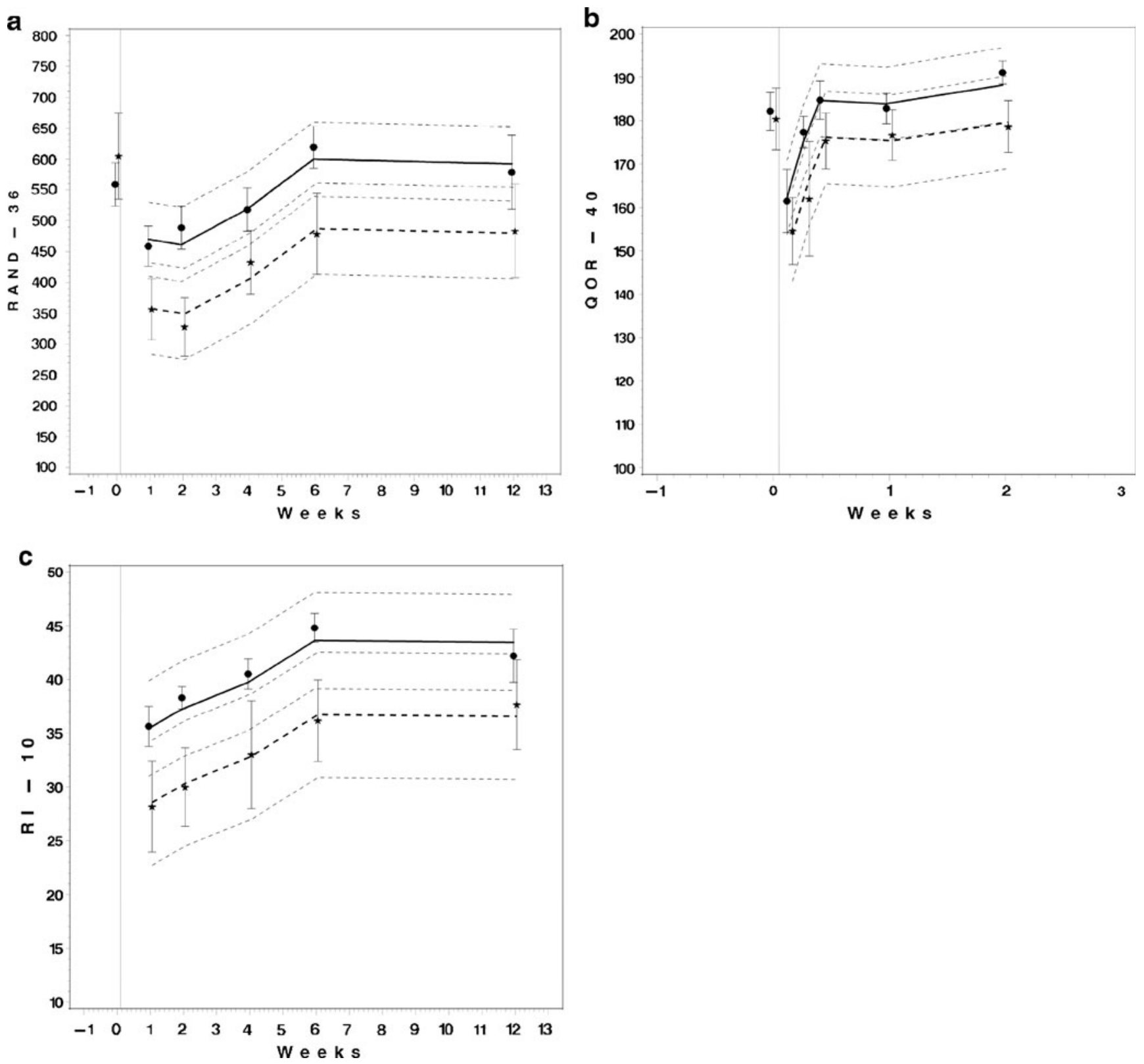

Fig. 2 a, $\mathbf{b}$ and $\mathbf{c}$. The mean levels of the questionnaire scores after surgery by treatment group. The symbols indicate the observed mean and the vertical bars indicate \pm one standard deviation. The thick lines indicate the estimated mean profiles and the thin, short dashed lines indicate the appropriate $95 \%$ confidence bands using a linear mixed model that accounts for the baseline value. Women in the laparoscopic

hysterectomy group: dot, solid line. Women in the abdominal hysterectomy group: star, thick dashed line. RAND-36 RAND 36Item Short Form Health Survey, QoR-40 Quality of Recovery-40, RI10 Recovery Index-10. Note that the data fit very well to the parallelline model

carcinoma patients has not yet been unravelled, and cannot be concluded from the present study. The incidence of major complications in laparoscopic hysterectomy may, however, be highly reduced with experience of the surgeon. In Finland, the incidence of urinary tract injury in laparoscopic hysterectomy decreased from $1.4 \%$ to $0.7 \%$ in recent years [25]. Ureteral damage now happens in only $0.3 \%$ of cases, which seems comparable to abdominal

hysterectomy. Dependent on patient preference, the potential risks should be weighed against the improved quality of life after surgery in individual cases.

The conclusion from this small RCT was that laparoscopic hysterectomy was associated with better quality of life in the recovery period as compared to abdominal hysterectomy in women with atypical endometrial hyperplasia and endometrial carcinoma. 
Acknowledgements We thank J.M. (Hanny) Pijnenborg, MD, PhD for her careful review of the gynaecologic oncology aspects of the paper.

Conflicts of interest The authors report no conflicts of interest.

Open Access This article is distributed under the terms of the Creative Commons Attribution Noncommercial License which permits any noncommercial use, distribution, and reproduction in any medium, provided the original author(s) and source are credited.

\section{References}

1. Nieboer TE, Johnson N, Lethaby A et al (2009) Surgical approach to hysterectomy for benign gynaecological disease. Cochrane Database Syst Rev. 8:CD003677

2. Kluivers KB, Hendriks JC, Mol BW, Bongers MY, Bremer GL, de Vet HC, Vierhout ME, Brolmann HAM (2007) Quality of life and surgical outcome after total laparoscopic hysterectomy versus total abdominal hysterectomy for benign disease: a randomized, controlled trial. J Minim Invasive Gynecol 14:145-152

3. Kluivers KB, Johnson NP, Chien P, Vierhout ME, Bongers M, Mol BW (2008) Comparison of laparoscopic and abdominal hysterectomy in terms of quality of life: a systematic review. Eur J Obstet Gynecol Reprod Biol 136:3-8

4. Tozzi R, Malur S, Koehler C, Schneider A (2005) Analysis of morbidity in patients with endometrial cancer: is there a commitment to offer laparoscopy? Gynecol Oncol 97:4-9

5. Zullo F, Palomba S, Russo T et al (2005) A prospective randomized comparison between laparoscopic and laparotomic approaches in women with early stage endometrial cancer: a focus on the quality of life. Am J Obstet Gynecol 193:1344-1352

6. Fram KM (2002) Laparoscopically assisted vaginal hysterectomy versus abdominal hysterectomy in stage I endometrial cancer. Int $\mathrm{J}$ Gynecol Cancer 12:57-61

7. Zorlu CG, Simsek T, Seker Ari E (2005) Laparoscopy or laparotomy for the management of endometrial cancer. JSLS 9:442-446

8. Malzoni M, Tinelli R, Cosentino F, Perone C, Rasile M, Iuzzolino D, Malzoni C, Reich H (2009) Total laparoscopic hysterectomy versus abdominal hysterectomy with lymphadenectomy for earlystage endometrial cancer: a prospective randomized study. Gynecol Oncol 112:126-133

9. Kornblith AB, Huang HQ, Walker JL, Spirtos NM, Rotmensch J, Cella D (2009) Quality of life of patients with endometrial cancer undergoing laparoscopic international federation of gynecology and obstetrics staging compared with laparotomy: a Gynecologic Oncology Group study. J Clin Oncol 27(32):5337-5342

10. Mourits MJ, Bijen CB, Arts HJ, ter Brugge HG, van der Sijde R, Paulsen L, Wijma J, Bongers MY, Post WJ, van der Zee AG, de Bock GH (2010) Safety of laparoscopy versus laparotomy in early-stage endometrial cancer: a randomised trial. Lancet Oncol 11(8):763-771

11. Janda M, Gebski V, Brand A, Hogg R, Jobling TW, Land R, Manolitsas T, McCartney A, Nascimento M, Neesham D, Nicklin JL, Oehler MK, Otton G, Perrin L, Salfinger S, Hammond I, Leung Y, Walsh T, Sykes P, Ngan H, Garrett A, Laney M, Ng TY, Tam K, Chan K, Wrede CD, Pather S, Simcock B, Farrell R,
Obermair A (2010) Quality of life after total laparoscopic hysterectomy versus total abdominal hysterectomy for stage I endometrial cancer (LACE): a randomised trial. Lancet Oncol 11 (8):772-780

12. Zullo F, Palomba S, Falbo A, Russo T, Mocciaro R, Tartaglia E, Tagliaferri P, Mastrantonio P (2009) Laparoscopic surgery vs laparotomy for early stage endometrial cancer: long-term data of a randomized controlled trial. Am J Obstet Gynecol 200(3):296.e1-296.e9

13. de la Orden SG, Reza MM, Blasco JA, Andradas E, Callejo D, Pérez T (2008) Laparoscopic hysterectomy in the treatment of endometrial cancer: a systematic review. J Minim Invasive Gynecol 15:395-401

14. van der Zee KI, Sanderman R (1993) Het meten van de gezondheidstoestand met de Rand - 36, een handleiding. Noordelijk Centrum voor gezondheidsvraagstukken, Groningen

15. Kluivers KB, Hendriks JC, Mol BW, Bongers MY, Vierhout ME, Brölmann HA, de Vet HC (2008) Clinimetric properties of 3 instruments measuring postoperative recovery in a gynecologic surgical population. Surgery 144:12-21

16. Creutzberg CL, van Putten WL, Koper PC, Lybeert ML, Jobsen JJ, Wárlám-Rodenhuis CC, De Winter KA, Lutgens LC, van den Bergh AC, van de Steen-Banasik E, Beerman H, van Lent M (2000) Surgery and postoperative radiotherapy versus surgery alone for patients with stage-1 endometrial carcinoma: multicentre randomised trial. Lancet 355:1404-1411

17. Verbeke G, Molenberghs G (1997) Linear mixed models in practice: a SAS-oriented approach. Springer, New York

18. ASTEC study group, Kitchener H, Swart AM, Qian Q, Amos C, Parmar MK (2009) Efficacy of systematic pelvic lymphadenectomy in endometrial cancer (MRC ASTEC trial): a randomised study. Lancet 373:125-136

19. Benedetti PP, Basile S, Maneschi F (2008) Systematic pelvic lymphadenectomy vs. no lymphadenectomy in early-stage endometrial carcinoma: randomized clinical trial. J Natl Cancer Inst 100:1707-1716

20. Mourits MJ, Bijen CB, de Bock GH (2009) Lymphadenectomy in endometrial cancer. Lancet 373:1169

21. Nout RA, Smit VT, Putter H, Jürgenliemk-Schulz IM, Jobsen JJ, Lutgens LC, van der Steen-Banasik EM, Mens JW, Slot A, Kroese MC, van Bunningen BN, Ansink AC, van Putten WL (2010) PORTEC Study Group. Vaginal brachytherapy versus pelvic external beam radiotherapy for patients with endometrial cancer of high-intermediate risk (PORTEC-2): an open-label, noninferiority, randomised trial. Lancet 375:816-823

22. Eltabbakh GH, Mount SL (2006) Laparoscopic surgery does not increase the positive peritoneal cytology among women with endometrial carcinoma. Gynecol Oncol 100:361-364

23. Lim S, Kim HS, Lee KB, Yoo CW, Park SY, Seo SS (2008) Does the use of a uterine manipulator with an intrauterine balloon in total laparoscopic hysterectomy facilitate tumor cell spillage into the peritoneal cavity in patients with endometrial cancer? Int $\mathrm{J}$ Gynecol Cancer 18:1145-1149

24. Logani S, Herdman AV, Little JV, Moller KA (2008) Vascular "pseudo invasion" in laparoscopic hysterectomy specimens: a diagnostic pitfall. Am J Surg Pathol 32:560-565

25. Brummer TH, Seppälä TT, Härkki PS (2008) National learning curve for laparoscopic hysterectomy and trends in hysterectomy in Finland 2000-2005. Hum Reprod 23:840-845 